\title{
Características do Pelame de Vacas Holandesas em Ambiente Tropical: Um Estudo Genético e Adaptativo 1
}

\author{
Alex Sandro Campos Maia², Roberto Gomes da Silva ${ }^{3}$, Elaine Cristina Abaker Bertipaglia ${ }^{4}$
}

\begin{abstract}
RESUMO - As características do pelame (espessura da capa, comprimento médio dos pêlos, número de pêlos por unidade de área, densidade de massa dos pêlos, ângulo de inclinação dos pêlos com respeito a superfície da epiderme e diâmetro médio do pêlos) foram avaliadas em 973 vacas da raça Holandesa, entre novembro de 2000 e abril de 2001, numa área localizada $20 \mathrm{~cm}$ abaixo da coluna vertebral, no centro do tronco, tanto nas malhas brancas como nas negras. As amostras de pêlos foram obtidas com um alicate comum adaptado. O método da Máxima Verossimilhança Restrita (REML) foi usado para estimar os componentes de variância e covariância sob modelo animal, sendo empregado o sistema MTDFREML. Os resultados mostraram que as características do pelame preto são diferentes das do branco, quando os animais são criados em ambiente tropical. O pelame preto apresentou-se menos denso, com pêlos mais curtos e grossos devido à maior necessidade de perder calor, enquanto que, o pelame branco é mais denso e com pêlos mais compridos, oferecendo uma melhor proteção contra à radiação solar direta. A seleção de vacas predominantemente negras pode ser uma boa escolha para aumentar a resistência do gado Holandês às condições do ambiente tropical, principalmente à radiação solar, quando esses animais são criados a campo, devido a que a epiderme sob esse tipo de malhas é altamente pigmentada. Tal seleção pode ser facilmente realizada, considerando a alta herdabilidade $\left(\mathrm{h}^{2}=0,75\right)$ para a proporção de malhas negras. Esta seleção deve ser realizada no sentido de um pelame menos denso, com pêlos curtos e grossos favorecendo as perdas de calor sensível e calor latente.
\end{abstract}

Palavras-chaves: adaptação, bovinos, raça Holandesa, pelame, seleção

\section{Haircoat Traits in Holstein Cows in Tropical Environments: a Genetic and Adaptative Study}

\begin{abstract}
The traits of the haircoat (haircoat thickness, average hair lenght with respect skin surface, number of hair per unit area, hair mass density, hair angle and average hair diameter) were estimated in 973 Holstein cattle in the period between November 2000 and April of 2001. These traits were measured in an area $20 \mathrm{~cm}$ below the dorsal line in the center of the thorax, for the black as for the white haircoat. Hair samplings were performed with adapted pliers. The method of Restricted Maximum Likelihood (REML) was used to estimate the variance and covariance components under the Animal model, the program used being the MTDFREML. The results showed that traits of black haircoat were different from that of the white haircoat, when the animals are breed in a tropical environment. Black haircoat was less dense, with shorter and ticker hair fibres in order to ease the heat dissipation, while the white haircoat was denser with longer hairs, which favored protection against direct solar radiation. The selection of predominantly black cows would be a good choice under the strong solar radiation in tropical environment, because the pigmented skin under this type coat. Such a selection could be easily performed considering the high heritability $\left(h^{2}=0.75\right)$ of the proportion of black coat, and must consider a less dense haircoat with short and tick hairs to help the dissipation sensible and latent heat.

Key Words: adaptation, cattle, Holstein cattle, haircoat, selection

\section{Introdução}

As características morfológicas e a cor do pelame em bovinos são fatores importantes que afetam diretamente as trocas térmicas de calor sensível (convecção cutânea e radiação) e as perdas de calor latente (evaporação cutânea) para o ambiente. Vários

trabalhos relacionados com esse tema têm sido publicados, em especial aqueles que relacionam esses aspectos adaptativos com as trocas térmicas por radiação (Hutchinson \& Brown, 1969; Kovarik, 1973; Cena \& Monteith, 1975a; Gebremedhin et al., 1997; McArthur, 1987 e 1991; Silva et al., 2001, 2002) e com as perdas de calor latente que ocorrem na

${ }_{1}^{1}$ Financiada pela Fundação de Amparo à Pesquisa do Estado de São Paulo (FAPESP), proc. 00/03022-5.

2 Estudante de Doutorado em Produção Animal, Laboratório de Bioclimatologia, Departamento de Zootecnia, Faculdade de Ciências Agrárias e Veterinárias (FCAV), Universidade Estadual Paulista (UNESP), 14884-900 Jaboticabal, SP. E.mail: alexsan@fcav.unesp.br ${ }^{3}$ Professor Titular, Laboratório de Bioclimatologia, Departamento de Zootecnia, FCAV IUNESP, 14884-900 Jaboticabal, SP. E.mail: rgomes@fcav.unesp.br

${ }^{4}$ Estudante de Mestrado, Departamento de Medicina Veterinária Preventiva e Reprodução Animal, FCAVUNESP, 14884-900 Jaboticabal, SP. E.mail: elaineab@fcav.unesp.br
\end{abstract}


superfície cutânea (Silva 1999; Cena \& Monteith, 1975b; Holmes, 1989; McArthur, 1987).

É geralmente aceito que o pelame escuro apresenta maior absorção e menor reflexão da radiação térmica, resultando em maior estresse de calor para os animais. Entretanto, tem sido demostrado que os pelames claros apresentam maior penetração da radiação solar que os escuros (Cena \& Monteith, 1975a; Hutchinson \& Brown, 1969; Silva et al, 2001; 2002). Utilizando modelos de Cena \& Monteith (1975a), Maia et al. (2002) confirmaram que a quantidade de radiação efetivamente transmitida através da capa de pelame depende não somente da cor, mas em alto grau da sua estrutura física, principalmente do número de pêlos por unidade de área. Cena \& Monteith (1975b) e Holmes (1981) já haviam sugerido que animais com pelames mais espessos e densos apresentam maior dificuldade para eliminar calor latente via evaporação cutânea. Este problema seria tanto mais acentuado quanto maior fosse a espessura da capa.

Segundo Silva (1999), um bovino mais adequado para ser criado a campo aberto em regiões tropicais deve apresentar um pelame de cor clara com pêlos curtos, grossos, medulados e bem assentados, sobre uma epiderme altamente pigmentada. Tais características físicas do pelame favoreceriam tanto a convecção como a evaporação na superfície cutânea, ao passo que altos níveis de melanina na epiderme dariam a proteção necessária contra a radiação ultravioleta.

O presente trabalho teve como objetivo realizar uma avaliação adaptativa das características morfológicas do pelame de vacas Holandesas, associadas à adequação às condições meteorológica naturais do ambiente tropical, principalmente a radiação solar e, ao mesmo tempo genética, visando proporcionar subsídios para a escolha do melhor padrão morfológico a ser utilizado em programas de melhoramento genético, para aumentar a adaptação e, em última análise, a capacidade produtiva dessa raça em ambiente tropical.

\section{Material e Métodos}

Foram utilizadas 973 fêmeas da raça Holandesa de um rebanho comercial em Descalvado, estado de São Paulo, Brasil (2201' S latitude, 4753' W longitude e 856 metros de altitude), sendo novilhas, vacas em lactação, vacas secas e próximas de parir, com idade entre 2 e 13 anos, filhas de 793 vacas e 205 touros. As coletas de dados foram realizadas das 07:30 às 15:00 horas, entre novembro de 2000 e abril de 2001. A proporção de malhas negras em relação à área de superfície corporal foi determinada diretamente através de uma avaliação visual em ambos os lados de cada animal, sendo desconsiderados a cauda, cabeça e membros.

A espessura da capa ( $\mathrm{mm}$ ) foi medida in situ utilizando-se uma régua metálica provida de um cursor (Silva, 2000). As demais características foram avaliadas a partir de amostras de pêlos coletadas $20 \mathrm{~cm}$ abaixo da coluna vertebral, no centro do tronco, tanto nas malhas negras como nas brancas, utilizando-se um alicate adaptado conforme Lee (1953). Essas características foram as seguintes: comprimento médio dos pêlos, número de pêlos por unidade de área, densidade de massa dos pêlos, ângulo de inclinação dos pêlos em relação à superfície da epiderme e diâmetro médio dos pêlos.

Para determinação do comprimento médio dos pêlos ( $\mathrm{mm})$, foi tomada a média aritmética do comprimento dos dez maiores pêlos, eleitos através de uma análise visual da amostra e medidos com paquímetro, seguindo o procedimento recomendado por Udo (1978). O número de pêlos por unidade de área (pêlos/cm2) foi estimado através da contagem do número de pêlos da amostra, correspondente à área de $18 \mathrm{~mm} 2$ da abertura entre as mandíbulas do alicate. A densidade de massa $(\mathrm{g} / \mathrm{cm} 3)$ foi obtida através da pesagem dos pêlos de cada amostra em balança analítica. $\mathrm{O}$ ângulo de inclinação dos pêlos em relação à superfície da epiderme (graus) foi obtido através da fórmula: ângulo=arc sen[espessura/comprimento], conforme Silva (2000). Para o diâmetro médio dos pêlos ( $\mathrm{mm}$ ) foi considerada a média aritmética do diâmetro dos dez maiores pêlos de cada amostra. Um micrômetro digital Mitutoyo foi utilizado para realização dessas medidas.

Nas analises estatísticas foi utilizado o teste de hipótese multivariado $\mathrm{T}^{2}$, de Hotelling com o objetivo de comparar diferentes grupos de vetores de médias (Johnson \& Wichern, 1992; Silva, 2000). O T² é expresso como:

$$
\mathrm{T}^{2}=\frac{\mathrm{n}_{1} \mathrm{n}_{2}}{\mathrm{n}_{1}+\mathrm{n}_{2}}\left[\mathbf{m}_{\mathbf{1}}-\mathbf{m}_{2}\right]^{\prime} \mathbf{S}^{-1}\left[\mathbf{m}_{\mathbf{1}}-\mathbf{m}_{\mathbf{2}}\right]
$$

em que $n_{1}$ e $n_{2}$ referem-se ao número de observações nas variáveis dentro de cada grupo. Os vetores e são os estimadores dos vetores de médias populacionais e 
das p características do pelame preto e branco, respectivamente,

$$
\mathbf{m}_{1}=\frac{1}{\mathrm{n}_{1}} \mathbf{Y}_{\mathrm{nxp}} \cdot\left[\begin{array}{c}
1 \\
\ldots \\
1_{\mathrm{n}}
\end{array}\right] \quad \mathbf{m}_{2}=\frac{1}{\mathrm{n}_{2}} \mathbf{Y}_{\mathrm{nxp}} \cdot\left[\begin{array}{c}
1 \\
\ldots \\
1_{\mathrm{n}}
\end{array}\right]
$$

em que: $\mathbf{Y}_{\text {nxp }}$ é a matriz com os valores de cada variável observada dentro de cada grupo de dimensões nxp,

$$
\mathbf{Y}_{\mathrm{nxp}}=\left[\begin{array}{ccc}
\mathrm{Y}_{11} & \ldots & \mathrm{Y}_{1 \mathrm{p}} \\
\ldots & \ldots & \ldots \\
\mathrm{Y}_{\mathrm{n} 1} & \ldots & \mathrm{Y}_{\mathrm{np}}
\end{array}\right]
$$

Sendo $\mathrm{S}$ a matriz das estimativas das variâncias e covariâncias, obtidas como:

$$
\mathbf{S}=\frac{\left(\mathrm{n}_{1}-1\right) \mathbf{S}_{\mathbf{1}}+\left(\mathrm{n}_{2}-1\right) \mathbf{S}_{\mathbf{2}}}{\mathrm{n}_{1}+\mathrm{n}_{2}-2}
$$

em que: $S_{1}$ e $S_{2}$ são as matrizes com as estimativas das variâncias e covariâncias do grupo 1 e 2, respectivamente,

$$
\begin{aligned}
& \mathbf{S}_{\mathbf{1}}=\frac{1}{\mathrm{n}_{1}-1} \mathbf{Y}_{\mathrm{nxp}} \cdot\left[\mathbf{I}_{\mathrm{nxn}}-\left(\frac{1}{\mathrm{n}_{1}}\right)\left[\begin{array}{c}
1 \\
\ldots \\
1_{\mathrm{n}}
\end{array}\right]\left[\begin{array}{lll}
1 & \ldots & 1_{\mathrm{n}}
\end{array}\right]\right] \mathbf{Y}_{\mathrm{nxp}} \\
& \mathbf{S}_{2}=\frac{1}{\mathrm{n}_{2}-1} \mathbf{Y}_{\mathrm{nxp}}{ }^{\prime}\left[\mathbf{I}_{\mathrm{nxn}}-\left(\frac{1}{\mathrm{n}_{2}}\right)\left[\begin{array}{c}
1 \\
\ldots \\
1_{\mathrm{n}}
\end{array}\right]\left[\begin{array}{lll}
1 & \ldots & 1_{\mathrm{n}}
\end{array}\right]\right] \mathbf{Y}_{\mathbf{n x p}}
\end{aligned}
$$

em que: $\mathbf{I}_{\text {nxn }}$ é uma matriz identidade de dimensões nxn.

A hipótese nula considerada foi $\mathrm{H} 0: \mu_{1}=\mu_{2}$ e a sua alternativa $\mathrm{H} 1: \mu_{1} \neq \mu_{2}$. Rejeita-se $\mathrm{H} 0$ se $\mathrm{T}^{2}>\mathrm{c}^{2}$, sendo:

$$
\mathrm{c}^{2}=\frac{\left(\mathrm{n}_{1}+\mathrm{n}_{2}-2\right) \mathrm{p}}{\mathrm{n}_{1}+\mathrm{n}_{2}-\mathrm{p}-1} \mathrm{~F}_{\left.\mathrm{p} ; \mathrm{n}_{1}+\mathrm{n}_{2}-\mathrm{p}-1\right) ; \dot{a}}
$$

onde $\mathrm{F}$ é o valor tabelado com $v_{1}=\mathrm{p}$ e $v_{2}=(\mathrm{n} 1+$ $\left.\mathrm{n}_{2}-\mathrm{p}-1\right)$ graus de liberdade, ao nível a de significância.

O intervalo de confiança para a diferença média pode ser calculado no caso em que $\mathrm{H} 0$ for rejeitada, visando identificar as variáveis que levaram à rejeição. Assim:

Limite Inferior $=\mathbf{A}^{\prime}\left(\mathbf{m}_{\mathbf{1}}-\mathbf{m}_{\mathbf{2}}\right)-\mathrm{K}$

Limite Superior $=\mathbf{A}^{\prime}\left(\mathbf{m}_{1}-\mathbf{m}_{2}\right)+\mathrm{K}$ em que:A é uma matriz identidade de dimensões pxp e K é obtida como:

$$
\mathrm{K}=\left[\operatorname{Diag}(\mathbf{S})\left(\frac{\mathrm{n}_{1}+\mathrm{n}_{2}}{\mathrm{n}_{1} \mathrm{n}_{2}}\right) \mathrm{c}^{2}\right]^{1 / 2}\left[\begin{array}{c}
1 \\
\ldots \\
1_{\mathrm{p}}
\end{array}\right]
$$

Se o intervalo de confiança incluir zero, a variável em questão não difere entre os dois grupos.

Antes de proceder às análises de variância, foi verificada a condição de normalidade da distribuição de freqüência dos valores dos erros das variáveis com o uso do procedimento univariate do SAS (Statistical Analysis System, versão 6.10). Se o teste de Shapiro-Wilk não fosse significativo, procedia-se às análises, em caso contrário, era feita a transformação da família modificada de Box \& Cox, (1964)

$$
\mathrm{Y}^{\prime}=\begin{array}{ll}
\frac{\mathrm{Y}^{\ddot{\mathrm{e}}}-1}{\ddot{\mathrm{e}}^{\ddot{\mathrm{e}}-1}} & \text { se } \ddot{\mathrm{e}} \neq 0 \\
\dot{\mathrm{Y}} \ln \mathrm{Y} & \text { se } \ddot{\mathrm{e}}=0
\end{array}
$$

em que: $\dot{Y}$ é a média geométrica de Y (Box \& Cox, 1964; Drapper \& Smith 1998).

As análises de variância foram realizadas usando-se o SAS. Os modelos matemáticos utilizados são descritos em seguida. Para o modelo (1),

$$
\mathrm{Y}_{\mathrm{ijklm}}=\mathrm{a}+\mathrm{m}_{\mathrm{i}}+\mathrm{a}_{\mathrm{j}}+\mathrm{o}_{\mathrm{k}}+\mathrm{s}_{\mathrm{kl}}+\mathrm{b}_{1}\left(\mathrm{C}_{\mathrm{ijk}}\right)+\stackrel{\mathrm{a}}{\mathrm{ijklm}}_{\mathrm{i}}
$$

em que: $Y_{\mathrm{ijklm}}$ é a média da espessura da capa e do diâmetro médio dos pêlos pretos e brancos da mésima vaca, mi é o efeito fixo do i-ésimo mês de coleta de amostragem $(i=1, \ldots, 6) ; a_{j}$ é o efeito fixo da $\mathrm{j}$-ésima classe de idade $(\mathrm{j}=1, \ldots, 6) ; \mathrm{o}_{\mathrm{k}}$ é o efeito fixo da k-ésima origem do pai da vaca $(\mathrm{k}=1, \ldots, 3) ; \mathrm{s}_{\mathrm{kl}}$ é o efeito aleatório do l-ésimo pai dentro da k-ésima origem (para $\mathrm{k}=1,1=1, \ldots, 21$; para $\mathrm{k}=2,1=1, \ldots, 38$ e para $\mathrm{k}=3,1=1, \ldots, 146) ; \mathrm{b}_{1}$ é o coeficiente de regressão sobre o comprimento médio dos pêlos $\left(\mathrm{C}_{\mathrm{ijkl}}\right), \varepsilon_{\mathrm{ijklm}}$ é o resíduo, incluindo o erro aleatório e a é o intercepto. A média geral é estimada por:

$$
\tilde{i}=\hat{a}+\hat{b}_{1} \bar{C}
$$

em que: $\overline{\mathrm{C}}$ a média geral do comprimento dos pêlos.

O modelo (2) foi:

$$
\mathrm{Y}_{\mathrm{ijklm}}=\grave{i}+\mathrm{m}_{\mathrm{i}}+\mathrm{a}_{\mathrm{j}}+\mathrm{o}_{\mathrm{k}}+\mathrm{s}_{\mathrm{kl}}+\stackrel{\circ}{\mathrm{ijklm}}_{\mathrm{g}}
$$

em que: $Y_{i j k l m}$ é a média do comprimento dos pêlos, do número de pêlos por unidade de área ou do ângulo de inclinação dos pêlos com relação à superfície da epiderme dos pêlos pretos e brancos; $\mu$ à média geral e os demais efeitos são os mesmos do modelo1.

O modelo (3) foi:

$\mathrm{Y}_{\mathrm{ijklm}}=\mathrm{a}+\mathrm{m}_{\mathrm{i}}+\mathrm{a}_{\mathrm{j}}+\mathrm{o}_{\mathrm{k}}+\mathrm{s}_{\mathrm{kl}}+\mathrm{b}_{1}\left(\mathrm{C}_{\mathrm{ijk} k}\right)+\mathrm{b}_{2}\left(\mathrm{~N}_{\mathrm{ijk} \mathrm{k}}\right)+\mathrm{b}_{3}\left(\mathrm{D}_{\mathrm{ijk}}\right)+\mathrm{a}_{\mathrm{i} \mathrm{jkl}}$

em que $\mathrm{Y}_{\mathrm{ijklm}}$ é a média da densidade de massa dos pêlos pretos e brancos; $b^{2}$ é o coeficiente de 
regressão sobre a número de pêlos por unidade de área (Nijkl), b3 é o coeficiente de regressão sobre o diâmetro médio dos pêlos (Dijkl) e os demais termos foram definidos no modelo 1. A média geral é estimada por:

$$
\hat{\imath}=\hat{a}+\hat{b}_{1} \bar{C}+\hat{b}_{2} \bar{N}+\hat{b}_{3} \bar{D}
$$

em que: $\overline{\mathrm{N}}$ e $\overline{\mathrm{D}}$ são os valores médios do número de pêlos por unidade de área e do diâmetro médio dos pêlos, respectivamente sendo os demais termos definidos como anteriormente.

O modelo (4) foi:

$$
\mathrm{Y}_{\mathrm{ijk}}=\grave{\mathrm{i}}+\mathrm{o}_{\mathrm{i}}+\mathrm{s}_{\mathrm{ij}}+\stackrel{\circ}{\mathrm{ijk}}_{\mathrm{in}}
$$

em que: $Y_{i j k}$ é a média da proporção de malhas negras, os demais termos são iguais aos do modelo 1 e 2 .

O método da Máxima Verossimilhança Restrita (REML) foi usado para estimar os componentes de variância e covariância sob modelo animal, sendo empregado o sistema MTDFREML (Boldman et al., 1993). Os valores iniciais das estimativas de variância foram estimados por meio do procedimento de modelos mistos do SAS, tendo como base os modelos anteriormente citados e levando-se em conta somente os efeitos fixos significativos. O modelo animal utilizado como base foi:

$$
\mathrm{Y}_{\mathrm{ijklm}}=\mathrm{a}+\mathrm{g}_{\mathrm{i}}+\mathrm{m}_{\mathrm{j}}+\mathrm{a}_{\mathrm{k}}+\mathrm{o}_{1}+\mathrm{b}_{1}\left(\mathrm{C}_{\mathrm{ijkl}}\right)+\mathrm{b}_{2}\left(\mathrm{~N}_{\mathrm{ijkl}}\right)+\mathrm{b}_{3}\left(\mathrm{D}_{\mathrm{ijkl}}\right)+\mathrm{a}_{\mathrm{ijklm}}
$$

em que: $g_{i}$ é o mérito genético do ith animal e os demais termos, conforme definidos anteriormente.

\section{Resultados e Discussão}

Na Tabela 1, são apresentadas média geral para proporção de malhas negras em relação à área de superfície corporal e as médias gerais para as características do pelame preto e branco.

Udo (1798) encontrou as seguintes médias 24,8 e $25,6 \mathrm{~mm} ; 39,3$ e $37,7 \mathrm{~mm} ; 1465$ e 1430 pêlos/cm2 para o comprimento, diâmetro e número de pêlos por unidade de área do pelame preto e branco, respectivamente, em vacas Holandesas na Holanda. Esses resultados quando comparados com os apresentados na Tabela 1 mostram claramente que, apesar de serem animais da mesma raça, as suas características do pelame são diferentes. As condições naturais de temperatura, umidade e, principalmente, a radiação solar às quais os animais são submetidos, quando introduzidos em ambiente tropical, certamente, são os fatores que levam a essas modificações. Menor comprimento dos pêlos verificado nos animais estudados, sem dúvida ocorre porque este aspecto facilita tanto a termólise convectiva como à evaporativa na superfície cutânea. Além de que o calor conduzido através dos pêlos individualmente é maior do que o conduzido pelo ar que os envolve; assim, quanto mais grossos forem essas fibras tanto maior será a quantidade de energia térmica conduzida através da capa. Portanto, maior diâmetro dos pêlos é uma vantagem em ambientes quentes, já que esses animais estão submetidos a uma carga térmica radiante bem superior do que a de regiões temperadas.

As vacas avaliadas apresentaram a média de $71,3 \%$ de sua superfície corporal coberta por malhas negras, valor esse que esteve próximo ao observado por Becerril et al. (1994) que estimou o valor de $74,4 \%$, utilizando a mesma metodologia. É importante citar que das 973 vacas avaliadas 384 são predominantemente negras ( $>90 \%$ de malhas negras) e somente 53 são predominantemente brancas ( $>90 \%$ de malhas brancas). Esses resultados indicam que os animais com maior área de sua superfície corporal coberta por malhas negras são mais vantajosos do ponto de vista adaptativo em regiões intertropicais, certamente essa vantagem deve-se a alta pigmentação da epiderme sob este tipo de malha. Como é sabido, a única função conhecida da melanina é a proteção contra a radiação ultravioleta. Sendo essa função fundamental para os animais que vivem nessas regiões a campo, onde a incidência da radiação ultravioleta é significativamente maior do que nas regiões ditas temperadas.

Pode-se observar na Tabela 1 que às médias das características do pelame branco foram diferentes das do pelame preto, principalmente para o número de pêlos por unidade de área. A estatística T2 mostrou que o vetor de médias observadas para as características do pelame preto diferiram estatisticamente $(\mathrm{P}<0,05)$ das do pelame branco, sendo o valor $\mathrm{T} 2=1009,83$ bem superior a c $2=12,63$. Os intervalos de confiança foram calculados, cujos valores encontram-se na Tabela 2.

Os intervalos de confiança das quatro primeiras características e da última não incluiram o valor zero, portanto, pode-se concluir que a espessura da capa do pelame preto difere estatisticamente da espessura da capa do pelame branco $(\mathrm{P}<0,05)$. O mesmo ocorreu para o comprimento, número de pêlos por unidade de área, densidade de massa e diâmetro. No entanto, Udo (1978) não observou o mesmo resultado em seu trabalho realizado em vacas Holandesas, onde a 
Tabela 1 - Médias das características morfológicas do pelame de vacas Holandesas

Table 1 - Means of the haircoat traits in Holstein cows

\begin{tabular}{|c|c|c|c|}
\hline $\begin{array}{l}\text { Características } \\
\text { Traits } \\
\end{array}$ & $\mathrm{n}^{1}$ & 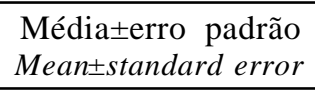 & $\begin{array}{l}\text { Desvio padrão } \\
\text { Standard deviation }\end{array}$ \\
\hline Proporção de malhas negras & 973 & $71,13 \pm 0,920$ & 28,72 \\
\hline \multicolumn{4}{|l|}{ Proportion of black cover } \\
\hline \multicolumn{4}{|l|}{ Espessura da capa $(\mathrm{mm})$ : } \\
\hline \multicolumn{4}{|l|}{ Haircoat thickness } \\
\hline Preto (black) & 973 & $2,35 \pm 0,014$ & 0,425 \\
\hline Branco (white) & 973 & $2,75 \pm 0,014$ & 0,438 \\
\hline \multicolumn{4}{|c|}{ Comprimento médio dos pêlos $(\mathrm{mm})$ : } \\
\hline \multicolumn{4}{|c|}{ Average hair length } \\
\hline Preto (black) & 973 & $12,05 \pm 0,100$ & 3,144 \\
\hline Branco (white) & 973 & $14,26 \pm 0,110$ & 3,570 \\
\hline \multicolumn{4}{|l|}{ Número de pêlos (pêlos $/ \mathrm{cm}^{2}$ ): } \\
\hline \multicolumn{4}{|l|}{ Number of hair } \\
\hline Preto (black) & 973 & $932 \pm 12$ & 381 \\
\hline Branco (white) & 973 & $1309 \pm 13$ & 403 \\
\hline \multicolumn{4}{|c|}{ Densidade de massa dos pêlos $\left(\mathrm{g} / \mathrm{cm}^{3}\right)$ : } \\
\hline \multicolumn{4}{|l|}{ Hair mass density } \\
\hline Preto (black) & 973 & $0,077 \pm 0,0012$ & 0,0366 \\
\hline Branco (white) & 973 & $0,092 \pm 0,0014$ & 0,0439 \\
\hline \multirow{2}{*}{\multicolumn{4}{|c|}{$\begin{array}{l}\text { Ângulo de inclinação dos pêlos (graus): } \\
\text { Hair angle }\end{array}$}} \\
\hline & & & \\
\hline Preto (black) & 973 & $11,83 \pm 0,097$ & 3,038 \\
\hline Branco (white) & 973 & $11,64 \pm 0,090$ & 2,802 \\
\hline \multicolumn{4}{|l|}{ Diâmetro médio dos pêlos $(\mathrm{mm})$ : } \\
\hline \multicolumn{4}{|l|}{ Average hair diameter } \\
\hline Preto (black) & 973 & $63,62 \pm 0,190$ & 5,944 \\
\hline Branco (white) & 973 & $60,53 \pm 0,180$ & 5,628 \\
\hline
\end{tabular}

${ }^{1}$ número de observações (number of observation).

Tabela 2 - Intervalos de confiança para diferença média entre características do pelame de vacas Holandesas Table 2 - Confidence interval for differences mean between the haircoat traits in Holstein cows

\begin{tabular}{|c|c|c|c|}
\hline \multirow[t]{2}{*}{$\begin{array}{l}\text { Características } \\
\text { Traits }\end{array}$} & \multicolumn{2}{|c|}{$\begin{array}{l}\text { Intervalo de confiança } \\
\text { Confidence interval }\end{array}$} & \multirow[t]{2}{*}{$\begin{array}{l}\text { Significância } \\
\text { Significance }\end{array}$} \\
\hline & $\begin{array}{l}\text { Limite inferior } \\
\text { Lower limit } \\
\end{array}$ & $\begin{array}{c}\text { Limite superior } \\
\text { Upper limit }\end{array}$ & \\
\hline $\begin{array}{l}\text { Espessura da capa } \\
\text { Haircoat thickness }\end{array}$ & $-0,4635$ & $-0,3244$ & $*$ \\
\hline $\begin{array}{l}\text { Comprimento médio dos pêlos } \\
\text { Average hair length }\end{array}$ & $-2,7395$ & $-1,6544$ & $*$ \\
\hline $\begin{array}{l}\text { Número de pêlos por unidade de área } \\
\text { Number of hair per unit area }\end{array}$ & $-439,2853$ & $-312,7147$ & $*$ \\
\hline $\begin{array}{l}\text { Densidade de massa do pelame } \\
\text { Hair mass density }\end{array}$ & $-0,0214$ & $-0,0085$ & $*$ \\
\hline $\begin{array}{l}\text { Ângulo de inclinação dos pêlos } \\
\text { Hair angle }\end{array}$ & $-0,2826$ & 0,6586 & ns \\
\hline $\begin{array}{l}\text { Diâmetro médio dos pêlos } \\
\text { Average hair diameter }\end{array}$ & 2,1768 & 4,0451 & $*$ \\
\hline
\end{tabular}

* Significativo $(\mathrm{P}<0,05)$ (significant $[P<.05]$.

ns Não significativo $(P>0,05)$ (not significant [P. 05]). 
média das características do pelame preto não diferiu estatisticamente da média das do pelame branco. As condições climáticas na Holanda verificado por Udo (1978) eram amenas, com temperatura média anual de $9,5^{\circ} \mathrm{C}$, enquanto que, a temperatura média na região de Descalvado é em torno de $22^{\circ} \mathrm{C}$.

Em um ambiente caracterizado por altas temperaturas, como de uma região tropical, a elevada incidência da radiação solar, certamente é o fator climático decisivo para que ocorram tais modificações nas propriedades físicas do pelame. Silva e LaScala (2001) determinaram a refletividade (r) e transmissividade (t) com um espectro - radiômetro LI-COR, modelo LI - 1800 para o comprimento de onda entre 300 e $850 \mathrm{~nm}$, tanto da epiderme sob as malhas negras como a das brancas, a partir de amostras de couros retiradas de animais recémmortos da raça Holandesa sendo a absortância (a) estimada por diferença $(\mathrm{a}=1-\mathrm{r}-\mathrm{t})$. Esses valores encontram-se na Figura 1.

Os resultados apresentados na Figura 1 concordam de, modo geral com o esperado através da literatura. A epiderme despigmentada apresentou coeficiente de transmissão consideravelmente mais elevado do que a epiderme pigmentada. Infere-se, portanto, que os maiores valores para o comprimento e principalmente para o número de pêlos por unidade de área observados no pelame branco dos animais avaliados devem-se à maior necessidade de prote-

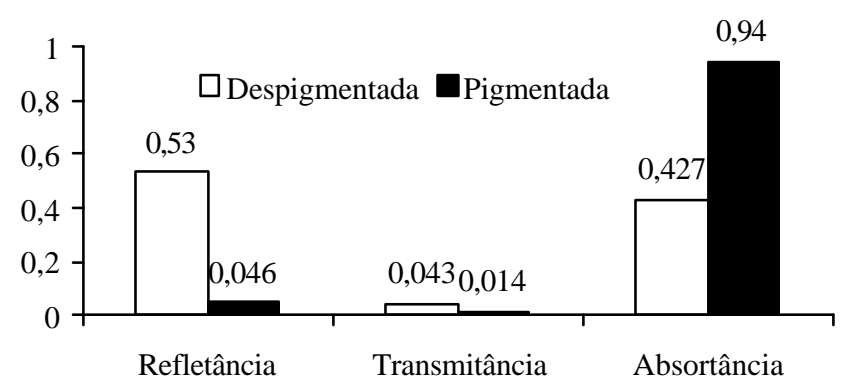

Figura 1 - Valores da refletância, transmitância e absortância da radiação solar da superfície da epiderme despigmentada e pigmentada de bovinos da raça Holandesa para faixa de comprimento de onda entre 300-850 nm.

Figure 1 - Value of reflectance, transmittance and absorptance of the solar radiation of the nonpigmented and pigmented skin surface in Holstein cattle for wavelength between 300 to $850 \mathrm{~nm}$. ção contra a radiação solar direta, devido a que a epiderme coberta pelas malhas brancas na raça Holandesa é despigmentada; nessas áreas, os pêlos são mais compridos e numerosos, constituindo uma barreira que reduz a penetração dos raios solares até a epiderme. Davis \& Birkebak (1974) e Gebremedhin et al. (1997) sugeriram também a importância do número de pêlos por unidade de área para a proteção da epiderme contra a radiação ultravioleta. Por outro lado, a melanina presente nos pêlos e na epiderme nas malhas negras confere uma proteção natural contra a radiação ultravioleta, porém há maior necessidade de perda de calor, uma vez que estas áreas absorvem maior quantidade de radiação térmica (Figura 1). Portanto, pêlos mais curtos, mais grossos e menos numerosos é uma vantagem neste tipo de malhas por oferecerem menor resistência à termólise por convecção e por evaporação que ocorre na superfície cutânea.

Nas Tabelas 3 e 4, são apresentadas as análises de variância para as características do pelame preto e branco, respectivamente. O efeito de mês foi significativo para todas as características, indicando haver forte variação, conforme o mês em que são coletadas. O mesmo foi observado por Udo (1978), Pinheiro (1996) e Silva et al. (1988). Nas Tabelas 5 e 6, encontram-se as médias das características do pelame preto e branco, respectivamente, conforme o mês de coleta, classe de idade e por origem do pai.

A respeito do efeito da idade, verifica-se na Tabela 5 que as vacas com idade superior a oito anos têm menor média para o comprimento, número de pêlos por unidade de área e maior para o diâmetro, apesar dessas duas últimas características não terem sido significativas $(\mathrm{P}>0,05)$. Esses resultados sugerem que está ocorrendo uma seleção inconsciente do rebanho na direção de vacas com pelame menos denso, com pêlos curtos e grossos nas áreas cobertas pelas malhas negras.

Constata-se nas Tabelas 5 e 6 tendência de os animais mais velhos terem menor quantidade de pêlos por unidade de área. Isto poderia dever-se ao fato de que o número de folículos por unidade de área diminuí com a idade (Turner et al., 1962), devido ao crescimento e expansão da epiderme, sem alteração no número de folículos. Todavia, é importante destacar que, quanto menor o número de pêlos por unidade de área, mais facilmente o vento penetra na capa, removendo o ar aprisionado entre os pêlos e favorecendo a transferência térmica. Essa característica é uma 
vantagem nas áreas de epiderme cobertas pelas malhas negras, mas o mesmo não se pode dizer das malhas brancas, devido à sua epiderme despigmentada, onde menor densidade de pêlos ofereceria menor proteção contra a radiação solar direta.

A análise de variância não detectou efeito significativo da origem do pai da vaca, exceto para o diâmetro médio dos pêlos pretos. Entretanto, as filhas de touros nascidos no Brasil apresentaram médias menores para as características do pelame preto (Tabela 5), quando comparadas com as filhas de pais nascidos no Canadá e nos Estados Unidos.

A estatística multivariada do $\mathrm{T}^{2}$ mostrou que o vetor médio das características do pelame preto das vacas filhas de touros nacionais diferiu estatistica- mente $(\mathrm{P}<0,05)$ do vetor médio das vacas filhas de touros importados (Canadá + Estados Unidos). O mesmo não aconteceu para o pelame branco, cuja diferença não foi significativa $(\mathrm{P}>0,05)$. Este resultado mostra que as vacas que são filhas dos touros nacionais, possuem de certa forma, características de pelame preto mais apropriadas ao ambiente tropical.

As estimativas de herdabilidade e as correlações genéticas e ambientais entre as características do pelame preto e branco encontram-se nas Tabelas 7 e 8 , respectivamente.

Os coeficientes $h^{2}$ não foram altos para o pelame preto, exceto para o diâmetro, indicando que a maior parte da variação nessas características é devida a fatores genéticos não aditivos e ambientais. O contrário

Tabela 3 - Quadrados médios das características do pelame preto em vacas Holandesa Table 3 - Mean squares of the traits of the black haircoat of Holstein cattle

\begin{tabular}{|c|c|c|c|c|c|c|c|}
\hline $\begin{array}{l}\text { Fonte } \\
\text { Source }\end{array}$ & $\begin{array}{l}\text { g.1 } \\
d f\end{array}$ & $E^{1}$ & $C^{2}$ & $\mathrm{~N}^{3}$ & $\mathrm{M}^{4}$ & $A^{5}$ & $\mathrm{D}^{6}$ \\
\hline Mês (Month) & 5 & $3,19^{* *}$ & $212,20^{* *}$ & $613691^{* *}$ & $0,0047^{* *}$ & $293,42^{* *}$ & $470,64^{* *}$ \\
\hline Idade $(A g e)$ & 5 & $0,25^{\mathrm{ns}}$ & $28,62^{* *}$ & $101694^{\mathrm{ns}}$ & $0,0024^{* *}$ & $10,83^{\mathrm{ns}}$ & $33,86^{\mathrm{ns}}$ \\
\hline Origem (Origin) & 2 & $0,08^{\text {ns }}$ & $9,50^{\mathrm{ns}}$ & $224710^{\mathrm{ns}}$ & $0,0006^{\mathrm{ns}}$ & $0,98^{\text {ns }}$ & $276,01^{* *}$ \\
\hline Pai (Or) Sire (Or) & 202 & $0,14^{*}$ & $9,61^{* *}$ & $147346^{\mathrm{ns}}$ & $0,0006^{\mathrm{ns}}$ & $7,81^{*}$ & $72,78^{* *}$ \\
\hline \multicolumn{8}{|c|}{ Regressão (Regression) } \\
\hline$b_{1}$ & 1 & $35,07^{* *}$ & - & - & $0,0171^{* *}$ & - & $1208,1^{* *}$ \\
\hline$b_{2}$ & 1 & - & - & - & $0,3337^{* *}$ & - & - \\
\hline $\mathrm{b}_{3}$ & 1 & - & - & - & $0,0217^{* *}$ & - & - \\
\hline Resíduo (Error) & 755 & 0,12 & 7,41 & 127564 & 0,0006 & 6,76 & 19,75 \\
\hline
\end{tabular}

** significativo $(P<0,01)$ (significant $[P<.01] ;{ }^{*}$ significativo $(P<0,05)$ (significant $[P<.05]$ ).

ns não significativo $(P>0,05)$ (non significant $(P>0.05)$.

${ }^{1}$ Espessura da capa (haircoat thickness); ${ }^{2}$ Comprimento médio dos pêlos (Average hair length); ${ }^{3}$ Número de pêlos por unidade de área(number of hair per unit area); ${ }^{4}$ densidade de Massa dos pêlos (hair mass density); ${ }^{5}$ Angulo de inclinação dos pêlos(hair angle); ${ }^{6}$ Diâmetro médio dos pêlos (average hair diameter).

Tabela 4 - Quadrados médios das características do pelame branco em vacas Holandesa

Table 4 - Mean squares of the traits of the white haircoat of Holstein cattle

\begin{tabular}{|c|c|c|c|c|c|c|c|}
\hline $\begin{array}{l}\text { Fonte } \\
\text { Source }\end{array}$ & $\begin{array}{l}\mathrm{gl} \\
d f\end{array}$ & $\mathrm{E}^{1}$ & $\mathrm{C}^{2}$ & $\mathrm{~N}^{3}$ & $\mathrm{M}^{4}$ & $A^{5}$ & $\mathrm{D}^{6}$ \\
\hline Mês (Month) & 5 & $1,45^{* *}$ & $519,50^{* *}$ & $2783720^{* *}$ & $0,0091^{* *}$ & $207,00^{* *}$ & $99,91^{* *}$ \\
\hline Idade (Age) & 5 & $0,23^{*}$ & $36,89^{* *}$ & $440304^{* *}$ & $0,0013^{\mathrm{ns}}$ & $6,41^{\mathrm{ns}}$ & $257,22^{* *}$ \\
\hline Origem (Origin) & 2 & $0,18^{\mathrm{ns}}$ & $9,42^{\mathrm{ns}}$ & $58616^{\mathrm{ns}}$ & $0,0004 * *$ & $0,05^{\mathrm{ns}}$ & $17,93^{\text {ns }}$ \\
\hline Pai(Or) Sire(Or) & 202 & $0,30^{* *}$ & $19,98^{* *}$ & $212242^{* *}$ & $0,0022^{* *}$ & $11,73^{* *}$ & $85,75^{* *}$ \\
\hline \multicolumn{8}{|c|}{ Regressão (regression) } \\
\hline $\mathrm{b}_{1}$ & 1 & $25,41^{* *}$ & - & - & $0,0171^{* *}$ & - & $63,50^{*}$ \\
\hline$b_{2}$ & 1 & - & - & - & $0,2295^{* *}$ & - & - \\
\hline $\mathrm{b}_{3}$ & 1 & - & - & - & $0,0096^{* *}$ & - & - \\
\hline Resíduo (Error) & 755 & 0,08 & 5,68 & 98385 & 0,0004 & 4,45 & 12,93 \\
\hline
\end{tabular}

${ }^{* *}$ Significativo $(P<0,01)$ (significant $(P<.01) ;{ }^{*}$ significativo $(P<0,05)$ (significant $[P<.05]$ ).

ns Não significativo $(P>0,05)$ (not significant [P.05]).

${ }^{1}$ Espessura da capa (haircoat thickness); ${ }^{2}$ Comprimento médio dos pêlos (Average hair length); ${ }^{3}$ Número de pêlos por unidade de área(number of hair per unit area); ${ }^{4}$ Densidade de massa dos pêlos (hair mass density); ${ }^{5}$ Ângulo de inclinação dos pêlos (hair angle);, o que causará um estresse de calor maior sobre esses animais; 6 Diâmetro médio dos pêlos (average hair diameter). 
Tabela 5 - Médias estimadas por quadrados mínimos das características do pelame preto, conforme o mês de coleta, a idade da vaca e a origem do pai da vaca

Table 5 - Least squares means of the black coat traits, according to the month of sampling, the age class and origin of the cow's sire

\begin{tabular}{|c|c|c|c|c|c|c|c|}
\hline Efeitos (Effects) & $\mathrm{n}^{7}$ & $E^{1}$ & $C^{2}$ & $\mathrm{~N}^{3}$ & $\mathrm{M}^{4}$ & $\mathrm{~A}^{5}$ & $\mathrm{D}^{6}$ \\
\hline \multicolumn{8}{|l|}{ Mês de coleta } \\
\hline \multicolumn{8}{|l|}{ Month of sampling } \\
\hline Nov/00 (Nov) & 391 & $2,44^{\mathrm{a}}$ & $10,97^{\mathrm{C}}$ & $925^{\mathrm{ab}}$ & $0,071^{b}$ & $12,95^{\mathrm{a}}$ & $64,24^{\mathrm{a}}$ \\
\hline Dez/00 (Dec) & 229 & $2,28^{b}$ & $12,09^{c}$ & $863^{\mathrm{b}}$ & $0,081^{\mathrm{a}}$ & $11,24^{\mathrm{b}}$ & $64,24^{\mathrm{a}}$ \\
\hline Jan/01 (Jan) & 49 & $2,47^{\mathrm{a}}$ & $12,13^{b c}$ & $748^{\mathrm{b}}$ & $0,075^{\mathrm{ab}}$ & $12,21^{\mathrm{ab}}$ & $61,06^{\mathrm{bc}}$ \\
\hline Fev/01 (Feb) & 80 & $2,50^{\mathrm{a}}$ & $11,00^{\mathrm{c}}$ & $1018^{a}$ & $0,079^{a b}$ & $13,24^{\mathrm{a}}$ & $62,52^{\mathrm{ab}}$ \\
\hline Mar/01 (Mar) & 177 & $2,15^{\mathrm{c}}$ & $13,53^{\mathrm{b}}$ & $1004^{\mathrm{a}}$ & $0,087^{\mathrm{a}}$ & $9,88^{\mathrm{c}}$ & $59,72^{c}$ \\
\hline $\mathrm{Abr} / 01$ (Apr) & 47 & $1,84^{\mathrm{d}}$ & $15,56^{\mathrm{a}}$ & $873^{a b}$ & $0,087^{\mathrm{a}}$ & $8,02^{\mathrm{d}}$ & $58,60^{\mathrm{C}}$ \\
\hline \multicolumn{8}{|l|}{ Classe de idade } \\
\hline \multicolumn{8}{|l|}{ da vaca (anos) } \\
\hline \multicolumn{8}{|l|}{ Class of the } \\
\hline \multicolumn{8}{|l|}{ cow age (years) } \\
\hline$<2$ & 55 & $2,15^{\mathrm{a}}$ & $13,09^{a b}$ & $1048^{a}$ & $0,072^{b}$ & $10,30^{\mathrm{a}}$ & $60,11^{\mathrm{a}}$ \\
\hline $2-3,5$ & 350 & $2,26^{\mathrm{a}}$ & $12,02^{\mathrm{ab}}$ & $909^{a}$ & $0,074^{\mathrm{b}}$ & $11,03^{\mathrm{a}}$ & $60,19^{\mathrm{a}}$ \\
\hline $3,5-5$ & 272 & $2,37^{\mathrm{a}}$ & $12,41^{\mathrm{ab}}$ & $869^{a}$ & $0,068^{\mathrm{b}}$ & $11,45^{\mathrm{a}}$ & $61,15^{\mathrm{a}}$ \\
\hline $5-6,5$ & 152 & $2,35^{\mathrm{a}}$ & $13,88^{\mathrm{a}}$ & $887^{\mathrm{a}}$ & $0,075^{\mathrm{b}}$ & $11,15^{\mathrm{a}}$ & $61,41^{\mathrm{a}}$ \\
\hline $6,5-8$ & 96 & $2,22^{\mathrm{a}}$ & $12,72^{\mathrm{ab}}$ & $851^{\mathrm{a}}$ & $0,086^{\mathrm{ab}}$ & $10,83^{\mathrm{a}}$ & $62,47^{\mathrm{a}}$ \\
\hline$>8$ & 48 & $2,33^{\mathrm{a}}$ & $11,15^{\mathrm{b}}$ & $866^{\mathrm{a}}$ & $0,105^{\mathrm{a}}$ & $12,79^{\mathrm{a}}$ & $65,04^{\mathrm{a}}$ \\
\hline \multicolumn{8}{|l|}{ Origem do pai da vaca } \\
\hline \multicolumn{8}{|l|}{ Origin of the cow sire } \\
\hline Brasil (Brazil) & 81 & $2,24^{\mathrm{a}}$ & $12,25^{\mathrm{a}}$ & $808^{a}$ & $0,076^{\mathrm{a}}$ & $11,12^{\mathrm{a}}$ & $58,59^{\mathrm{c}}$ \\
\hline Canada (Canadian) & 181 & $2,28^{\mathrm{a}}$ & $12,48^{\mathrm{a}}$ & $976^{a}$ & $0,084^{\mathrm{a}}$ & $11,28^{a}$ & $64,38^{\mathrm{a}}$ \\
\hline Estados Unidos (USA) & 711 & $2,31^{\mathrm{a}}$ & $12,91^{\mathrm{a}}$ & $930^{\mathrm{a}}$ & $0,080^{\mathrm{a}}$ & $11,38^{a}$ & $62,20^{\mathrm{b}}$ \\
\hline
\end{tabular}

${ }^{1}$ Espessura da capa (haircoat thickness); ${ }^{2}$ Comprimento médio dos pêlos (Average hair length); ${ }^{3}$ Número de pêlos por unidade de área(number of hair per unit area); ${ }^{4}$ densidade de Massa dos pêlos (hair mass density); ${ }^{5}$ Angulo de inclinação dos pêlos (hair angle); ${ }^{6}$ Diâmetro médio dos pêlos (average hair diameter) e ${ }^{7}$ Número de Observações(number of observation). Médias seguidas da mesma letra, para cada efeito e dentro de cada coluna, não difere estatisticamente pelo teste Tukey $(P>0,05)$ (Means with the same superscript, for each effect and within each column, do not differ statistically by test Tukey P.05).

ocorreu para o pelame branco, exceto para o número de pêlos por unidade de área. $\mathrm{A} \mathrm{h}^{2}$ para espessura da capa do pelame foi inferior à encontrada por Silva et al. (1988) em vacas Jersey $\left(h^{2}=0,233\right)$, ao passo que foi superior para o comprimento médio dos pêlos $\left(h^{2}=0,081\right)$. Pinheiro (1996) estimou, em vacas Holandesas, valores nulos para espessura e para número de pêlos por unidade de área $(\mathrm{h} 2=0,00)$, sendo inferior ao do presente trabalho o obtido para o comprimento médio dos pêlos $\left(h^{2}=0,20\right)$.

Estes valores de $\mathrm{h}^{2}$ estimados por esta autora são, de certa maneira, surpreendentes, pois em razão do valor obtido para o comprimento, esperar-se-ia um valor pelo menos próximo para espessura, que é determinada pelo comprimento em boa proporção. Portanto, as $h^{2}$ estimadas no presente trabalho são mais condizentes com a realidade que as de Pinheiro (1996).

$\mathrm{O}$ valor de $\mathrm{h}^{2}$ para proporção de malhas negras foi de $0,75 \pm 0,076$ (variável transformada com $1=1,70$ ), inferior aos valores estimados por Briquet \& Lush (1947). Para a proporção de malhas brancas que foram $h^{2}=0,99$ e $h^{2}=0,92$. King et al. (1988) estimaram $\mathrm{h}^{2}=0,22$, valor considerado pequeno, porém estes autores agruparam os animais por categoria (branco, misto, negras). Becerril et al. (1993) encontraram valor $h^{2}=0,715$, para essa característica.

Os coeficientes de correlação genética entre as características do pelame preto indicaram que os fatores genéticos envolvidos que causam aumento no comprimento, na espessura, no número de pêlos por unidade de área e na densidade de massa foram os mesmos. Estas correlações sugerem que seleção para a diminuição do comprimento resultaria em seleção simultânea para menor espessura, menor número de pêlos por unidade de área e maior ângulo de inclinação o que é desejável em ambiente tropical, uma vez que tanto o comprimento como a espessura são características que devem ser selecionadas, negativamente, em climas quentes. Entretanto, o mesmo não se pode dizer das áreas cobertas pelas malhas brancas, onde a seleção deve ser realizada no sentido de um pelame mais denso e com pêlos compridos. 
Tabela 6 - Médias estimadas por quadrados mínimos das características do pelame branco, conforme o mês de coleta, a idade da vaca e a origem do pai da vaca

Table 6 - Least squares means of the white coat traits, according to the month of sampling, the age class and origin of the cow sire

\begin{tabular}{lccccccc}
\hline Efeitos (Effects) & $\mathrm{n}^{7}$ & $\mathrm{E}^{1}$ & $\mathrm{C}^{2}$ & $\mathrm{~N}^{3}$ & $\mathrm{M}^{4}$ & $\mathrm{~A}^{5}$ \\
\hline Nov/00 (Nov) & 391 & $2,83^{\mathrm{b}}$ & $13,26^{\mathrm{d}}$ & $1198^{\mathrm{c}}$ & $0,083^{\mathrm{b}}$ & $12,49^{\mathrm{a}}$ & $59,53^{\mathrm{a}}$ \\
Dez/00 (Dec) & 229 & $2,72^{\mathrm{c}}$ & $13,82^{\mathrm{d}}$ & $1213^{\mathrm{c}}$ & $0,095^{\mathrm{b}}$ & $11,53^{\mathrm{b}}$ & $60,72^{\mathrm{a}}$ \\
Jan/01 (Jan) & 49 & $3,01^{\mathrm{a}}$ & $15,29^{\mathrm{c}}$ & $1194^{\mathrm{c}}$ & $0,097^{\mathrm{b}}$ & $12,21^{\mathrm{ab}}$ & $61,15^{\mathrm{a}}$ \\
Fev/01 (Feb) & 80 & $2,87^{\mathrm{ab}}$ & $13,65^{\mathrm{d}}$ & $1404^{\mathrm{b}}$ & $0,095^{\mathrm{b}}$ & $12,40^{\mathrm{a}}$ & $59,13^{\mathrm{b}}$ \\
Mar/01 (Mar) & 177 & $2,64^{\mathrm{c}}$ & $17,23^{\mathrm{b}}$ & $1599^{\mathrm{a}}$ & $0,110^{\mathrm{a}}$ & $9,72^{\mathrm{c}}$ & $58,49^{\mathrm{b}}$ \\
Abr/01 (Apr) & 47 & $2,46^{\mathrm{d}}$ & $20,42^{\mathrm{a}}$ & $1306^{\mathrm{bc}}$ & $0,094^{\mathrm{b}}$ & $8,28^{\mathrm{c}}$ & $60,98^{\mathrm{a}}$
\end{tabular}

Classe de idade da

vaca (anos)

Class of the cow

age (years)

\begin{tabular}{|c|c|c|c|c|c|c|c|}
\hline$<2$ & 55 & $2,65^{\mathrm{a}}$ & $16,39^{\mathrm{ab}}$ & $1613^{\mathrm{a}}$ & $0,081^{\mathrm{b}}$ & $10,49^{a}$ & $54,34^{\mathrm{c}}$ \\
\hline $2-3,5$ & 350 & $2,75^{\mathrm{a}}$ & $14,67^{\mathrm{c}}$ & $1412^{b}$ & $0,095^{\mathrm{a}}$ & $11,29^{\mathrm{a}}$ & $60,20^{\mathrm{ab}}$ \\
\hline $3,5-5$ & 272 & $2,79^{\mathrm{a}}$ & $15,93^{\mathrm{b}}$ & $1283^{b c}$ & $0,098^{\mathrm{a}}$ & $10,98^{\mathrm{a}}$ & $59,96^{\mathrm{a}}$ \\
\hline $5-6,5$ & 152 & $2,90^{\mathrm{a}}$ & $16,66^{\mathrm{a}}$ & $1267^{b c}$ & $0,093^{\mathrm{ab}}$ & $11,17^{\mathrm{a}}$ & $58,29^{b}$ \\
\hline $6,5-8$ & 96 & $2,81^{\mathrm{a}}$ & $14,83^{b c}$ & $1126^{c}$ & $0,101^{a b}$ & $11,89^{\mathrm{a}}$ & $62,99^{\mathrm{a}}$ \\
\hline$>8$ & 48 & $2,65^{\mathrm{a}}$ & $15,19^{b c}$ & $1215^{\mathrm{bc}}$ & $0,106^{\mathrm{ab}}$ & $10,80^{\mathrm{a}}$ & $64,24^{\mathrm{a}}$ \\
\hline Origem do pai da vaca & 81 & $2,85^{\mathrm{a}}$ & $16,03^{\mathrm{a}}$ & $1328^{\mathrm{a}}$ & $0,099^{\mathrm{a}}$ & $11,10^{\mathrm{a}}$ & $59,13^{\mathrm{a}}$ \\
\hline $\begin{array}{l}\text { Origin of the cow sire } \\
\text { Brasil (Brazil) }\end{array}$ & & & & & & & \\
\hline Canada (Canadian) & 181 & $2,69^{\mathrm{a}}$ & $15,13^{\mathrm{a}}$ & $1292^{\mathrm{a}}$ & $0,096^{\mathrm{a}}$ & $11,08^{\mathrm{a}}$ & $60,48^{a}$ \\
\hline Estados Unidos (USA) & 711 & $2,73^{\mathrm{a}}$ & $15,67^{\mathrm{a}}$ & $1338^{\mathrm{a}}$ & $0,093^{\mathrm{a}}$ & $11,13^{\mathrm{a}}$ & $60,39^{a}$ \\
\hline
\end{tabular}

${ }^{1}$ Espessura da capa (haircoat thickness); ${ }^{2}$ Comprimento médio dos pêlos (Average hair length); ${ }^{3}$ Número de pêlos por unidade de área(number of hair per unit area); ${ }^{4}$ densidade de Massa dos pêlos (hair mass density); ${ }^{5}$ Angulo de inclinação dos pêlos (hair angle); ${ }^{6}$ Diâmetro médio dos pêlos (average hair diameter) e ${ }^{7}$ Número de Observações (number of observation). Médias seguidas da mesma letra, para cada efeito e dentro de cada coluna, não difere estatisticamente pelo teste Tukey $(P>0,05)$ (Means with the same superscript, for each effect and within each column, do not differ statistically by test Tukey P 0.05 )

Tabela 7 - Coeficientes de correlação genética entre as características do pelame preto acima da diagonal e abaixo os coeficientes de correlação ambiental. Na diagonal, estão as herdabilidades

Table 7 - Genetic correlation coefficients among traits of black coat (upper diagonal) and environmental correlation (below diagonal). Heritabilities ( \pm s.e) on the diagonal

\begin{tabular}{lcccccc}
\hline & $\mathrm{E}^{1}$ & $\mathrm{C}^{2}$ & $\mathrm{~N}^{3}$ & $\mathrm{M}^{4}$ & $\mathrm{~A}^{5}$ & $\mathrm{D}^{6}$ \\
\hline $\mathrm{E}$ & $0,08 \pm 0,05$ & $0,78 \pm 0,19$ & $042 \pm 0,37$ & $0,19 \pm 0,54$ & $-1,00 \pm 0,68$ & $-0,06 \pm 0,22$ \\
$\mathrm{C}$ & 0,164 & $0,37 \pm 0,08$ & $0,24 \pm 0,25$ & $0,43 \pm 0,22$ & $-0,87 \pm 0,11$ & $-0,24 \pm 0,12$ \\
$\mathrm{~N}$ & 0,051 & 0,257 & $0,11 \pm 0,037$ & $0,06 \pm 0,41$ & $-0,07 \pm 0,37$ & $0,34 \pm 0,19$ \\
$\mathrm{M}$ & $-0,417$ & 0,329 & 0,545 & $0,14 \pm 0,07$ & $-0,06 \pm 0,37$ & $0,64 \pm 0,15$ \\
$\mathrm{~A}$ & 0,902 & $-0,557$ & $-0,083$ & $-0,399$ & $0,10 \pm 0,07$ & $-0,25 \pm 0,18$ \\
$\mathrm{D}$ & $-0,105$ & $-0,651$ & 0,110 & 0,167 & 0,020 & $0,75 \pm 0,07$ \\
\hline
\end{tabular}

${ }^{1}$ Espessura da capa(haircoat thickness); ${ }^{2}$ Comprimento médio dos pêlos(Average hair length); ${ }^{3}$ Número de pêlos por unidade de área (number of hairs per unit area); ${ }^{4}$ densidade de Massa dos pêlos (hair mass density); ${ }^{5}$ Angulo de inclinação dos pêlos (hair angle); ${ }^{6}$ Diâmetro médio dos pêlos (average hair diameter). 
Tabela 8 - Coeficientes de correlação genética entre as características do pelame branco acima da diagonal e abaixo os coeficientes de correlação ambiental. Na diagonal estão as herdabilidades

Table 8 - Genetic correlation coefficients between the traits of white coat (upper diagonal) and environmental correlation (below diagonal). Heritabilities ( \pm s.e) on the diagonal

\begin{tabular}{lcccccc}
\hline & $\mathrm{E}^{1}$ & $\mathrm{C}^{2}$ & $\mathrm{~N}^{3}$ & $\mathrm{M}^{4}$ & $\mathrm{~A}^{5}$ & $\mathrm{D}^{6}$ \\
\hline $\mathrm{E}$ & $0,59 \pm 0,07$ & $0,46 \pm 0,08$ & $0,10 \pm 0,13$ & $-0,26 \pm 0,08$ & $0,96 \pm 0,02$ & $-0,06 \pm 0,10$ \\
$\mathrm{C}$ & 0,352 & $0,69 \pm 0,07$ & $0,37 \pm 0,11$ & $0,32 \pm 0,079$ & $-0,72 \pm 0,055$ & $0,27 \pm 0,09$ \\
$\mathrm{~N}$ & $-0,209$ & 0,100 & $0,12 \pm 0,04$ & $-0,09 \pm 0,104$ & $-0,21 \pm 0,133$ & $0,05 \pm 0,11$ \\
$\mathrm{M}$ & $-0,4072$ & 0,371 & $-0,098$ & $0,79 \pm 0,058$ & $-0,32 \pm 0,087$ & $0,28 \pm 0,08$ \\
$\mathrm{~A}$ & 0,855 & $-0,635$ & $-0,155$ & $-0,361$ & $0,46 \pm 0,073$ & $-0,17 \pm 0,10$ \\
$\mathrm{D}$ & $-0,054$ & $-0,103$ & 0,111 & 0,010 & 0,018 & $0,70 \pm 0,06$ \\
\hline
\end{tabular}

${ }^{1}$ Espessura da capa(haircoat thickness); ${ }^{2}$ Comprimento médio dos pêlos(Average hair length); ${ }^{3}$ Número de pêlos por unidade de área (number of hairs per unit area); ${ }^{4}$ densidade de Massa dos pêlos (hair mass density); ${ }^{5}$ Angulo de inclinação dos pêlos (hair angle) e ${ }^{6}$ Diâmetro médio dos pêlos (average hair diameter).

Porém, torna-se impraticável a seleção de animais com os dois tipos de pelame simultaneamente. Por isso, a seleção dever ser realizada no sentido de vacas predominantemente negras ou brancas.

Se a seleção for realizada na direção de vacas com pelame preto, esses animais têm melhor proteção contra a radiação solar, embora eles passem a absorver maior quantidade de energia térmica (Figura 1), de modo que a temperatura da superfície cutânea será mais elevada, o que causará maior estresse de calor sobre esses animais, conforme demostraram Hansen (1990), Hillman et al. (2001) e Silva et al. (2001). No entanto, a termólise convectiva será favorecida pelo maior gradiente entre a temperatura da superfície cutânea e a do ar. Por outro lado, se a seleção for na direção de vacas com pelame branco, estes apresentarão grande área de epiderme despigmentada e, portanto, terão reduzida proteção contra a radiação $U V$, o que constituíra um grave problema para os mesmos em condições de campo (Silva et al., 2001).

Dito isso, a seleção de vacas com pelame predominantemente negro pode ser uma boa escolha para aumentar a resistência do gado Holandês às condições do ambiente tropical, principalmente a forte radiação solar quando esses animais são criados a campo aberto. Tal seleção poderia ser facilmente realizada, considerando a alta herdabilidade $\left(h^{2}=0,75\right)$ para a proporção de malhas negras. Esta seleção deve ser realizada no sentido de um pelame menos denso, com pêlos curtos e grossos para favorecer as perdas de calor latente e calor sensível. No entanto, os coeficientes de $\mathrm{h}^{2}$ não foram altos para as características do pelame preto. Com isso, as mudanças genéticas como resultado de seleção individual, dependerá da intensidade da seleção, devendo ser a mais intensa possível, para que as mesmas sejam fixadas na população em poucas gerações.

\section{Conclusões}

As características do pelame das vacas Holandesa observadas indicam um boa adequação às condições do ambiente tropical. O pelame peto difere do pelame branco no mesmo animal, nesta raça, sendo esses aspectos diferentes daqueles observados para a mesma raça em animais criados em clima temperado.

Os animais predominantemente negros são melhor protegidos contra a radiação solar que os predominantemente brancos. Portanto, a seleção deste tipo de vaca pode ser uma boa escolha para aumentar a resistência do gado Holandês às condições do ambiente tropical, principalmente a forte radiação solar quando esses animais são criados a campo aberto. No entanto, esta seleção deve ser realizada no sentido de um pelame menos denso com pêlos bem assentados, grossos e curtos, para favorecer as perdas de calor latente e sensível através da camada de pêlos.

\section{Agradecimento}

À Fundação de Amparo a Pesquisa do Estado de São Paulo (FAPESP), proc. 00/03022-5, pelo apoio financeiro. À Srta. Angela Arduíno, técnica do Laboratório de Bioclimatologia da FCAV, campus de Jaboticabal, pela inestimável colaboração. À Agrundus S.A., pela disponibilidade do rebanho.

\section{Literatura Citada}


BECERRIL, C.M.; WILCOX, C.J.; WIGGANS, G.R. et al. Transformation of measurements percentage of white coat color for Holsteins and estimation of heritability.Journal of Dairy Science, v.77, n.9, p.2651-2657, 1994.

BOLDMAN, K.G.; KRIESE, L.A.; Van VLECK, L.D. A manual for use of MTDFREML: A set of programs to obtain estimates of variances and covariances (DRAFT).Lincoln: Department of Agriculture, Agricultural Research Service, 1993. 120p.

BOX, G.E.P.; COX, D.R. An analysis of transformations. Journal of Royal Statistical Society, série B, v.26, p.211-252, 1964.

BRIQUET, R.; LUSH, J.L. Heritability of amount of spotting in Holstein-Friesian cattle. Journal Hered, v.38, p.99, 1947.

CENA, K.; MONTEITH, J.L. Transfer processes in animal coats. I. Radiative transfer. Procedure Royal Society London, v.188, p.377-393, 1975a.

CENA, K.; MONTEITH, J.L. Transfer processes in animal coats. III. Water vapor diffusion.Procedure Royal Society London, v.188, p.413-423, $1975 b$.

DAVIS, L.B.; BIRKEBAK, R.C. On the transfer of energy in layers of fur.Biophysics of Journal, v.14, p.249-268, 1974.

DRAPPER, N. R.; SMITH, H.Applied regression analysis.3. ed. New York: Wiley, 1998. 436p.

GEBREMEDHIN, K.G.; NI, H.; HILLMAN, P.E. Modeling temperature profile and Heat flux through irradiated fur layer. Transactions of the ASAE, v.40, n.5, p.1441-1447,1997.

HANSEN, P.J. Effects of coat colour on physiological responses to solar radiation in Holsteins. Veterinary Record, v.127, n.13, p.333-334, 1990.

HILLMAN, P.E.; LEE, C.N. PARKHURST, A. Impact of hair color on thermoregulation of dairy cows to direct sunlight. In: ANNUAL INTERNATIONAL MEETING OF THE ASAE, 94., 2001, Sacramento. Proceedings... Sacramento: 2001.

HOLMES, C.W. A note on the protection provided by the hair coat or fleece of the animal against the thermal effects of simulated rain. Animal Production, v.32, p.225-226, 1981.

HUTCHINSON, J.C.D.; BROWN, G.D. Penetrance of cattle coats by radiation. Jounal of Applied Physiology, v.26, p.454-464, 1969.

JOHNSON, R. A.; WICHERN, D. W. Applied multivariate statistical analysis, 3 ed. Englewood cliffs: Prentice-Hall, Inc., 1992, $642 \mathrm{pp}$.

KING, V.L.; DENISE, S.K.; ARMSTRONG, D.V. et al. Effects of a hot climate on the performance of first lactation Holstein cows grouped by coat color. Journal of Dairy Science, v.71, p.1093-1096, 1988.

KOVARIK, M. Radiation penetrence of protective covers. Journal of Applied Physiology, v.35, p.562-563,1973.

LEE, D.H.K. Manual of field studies on heat tolerance of domestic animals. Roma: FAO, 1953. 161p.

MAIA, A. S. C.; SILVA, R.G.; BERTIPAGLIA, E.C.A. Genetic and environmental variation of the effective radiative properties of the coat in Holstein cattle. In: WORLD CONGRESS OF THE GENETIC APLLIED TO LIVESTOCK PRODUCTION, 7., 2002, Montpellier. Proceedings... Montpellier: 2002.

MCARTHUR, A.J. Thermal radiation exchange, convection and the storage of latent heat in animal coats. Agricultural Forest Meteorology, v.53, p.325-336, 1991.

PINHEIRO, M.G. Variação genética de características da capa externa de vacas da raça Holandesa em ambiente tropical.Ribeirão Preto: Faculdade de Medicina, 1996. 43p. Tese (Doutorado em Genética) - Faculdade de Medicina/ Universidade de São Paulo, 1996.

SILVA. R.G.; ARANTES NETO, J.G.; HOLTZ -FILHO, S.V. Genetic aspects of the variation of the sweating rate and coat traits of Jersey cattle. Brazilian Journal Genetics, v.11, p.335-347, 1988.

SILVA, R.G. Estimativa do balanço térmico por radiação em vacas Holandesas expostas ao sol e á sombra em ambiente tropical. Revista Brasileira de Zootecnia, v.28, n.6, p.14031411, 1999.

SILVA, R.G. Introdução à bioclimatologia animal. São Paulo: Nobel, 2000. 286p.

SILVA, R.G.; LA SCALA JR., N.; POCAY, P.L.B. Transmissão de radiação ultravioleta através do pelame e da epiderme de bovinos. Revista Brasileira de Zootecnia, v.30, n.6, p.1939-1947, 2001.

SILVA, R.G.; LA SCALA JR., N. Propriedades radiativas do pelame e da epiderme de bovinos. In: III CONGRESSO BRASILEIRO DE BIOMETEOROLOGIA, 7., 2001, Maringá. Anais... Maringá: 2001. (CD-ROM)

SILVA, R.G.; LA SCALA JR., N.; TONHATI, H. Radiative properties of the body surface of cattle and other animals. Transactions of the ASAE, 2002 (no prelo).

SAS INSTITUTE.Suser's guide: Statistics. Version 6, 10.ed. Cary: 1995. 295p.

TURNER, H.G.; NAY, T.; FRENCH, G.T. The hair follicle population of cattle in relation to breed in body weight. Australian Journal of Agricultural Research, v.13, p.960-973, 1962.

UDO, H.M.J. Hair coat characteristics in Friesian heifers in the Netherlands and Kenya. Wageningen: Meded. Landbouwhogeschool Wageningen, 1978. 135p.

Recebido em: 24/07/02

Aceito em: 09/01/03 